\title{
PANORAMA DO USO DE MÍDIAS SOCIAIS NA GESTÃO DO CONHECIMENTO ORGANIZACIONAL
}

\section{ARTIGO DE REVISÃO}

ALMEIDA, Isabela Eugenio ${ }^{1}$, ANDRADE, Emmanuel Paiva de ${ }^{2}$

ALMEIDA, Isabela Eugenio. ANDRADE, Emmanuel Paiva de. Panorama do uso de mídias sociais na gestão do conhecimento organizacional. Revista Científica Multidisciplinar Núcleo do Conhecimento. Ano 06, Ed. 05, Vol. 01, pp. 107-119. Maio de 2021. ISSN: 2448-0959, Link de acesso:

https://www.nucleodoconhecimento.com.br/administracao/conhecimentoorganizacional, DOI:

10.32749/nucleodoconhecimento.com.br/administracao/conhecimentoorganizacional

\section{RESUMO}

O uso de mídias sociais e outras tecnologias digitais na gestão do conhecimento organizacional vem se destacando na literatura recente, embora sua compreensão tenha se mostrado desafiadora. $O$ presente artigo tem como objetivo traçar um panorama da pesquisa atual a respeito dos usos das mídias sociais na gestão do conhecimento. Para tanto, foi realizada uma revisão da literatura que buscou artigos que relacionassem esses dois elementos. A partir desse levantamento, foi possível concluir que as mídias sociais têm sido usadas com sucesso ou têm potencial para

\footnotetext{
${ }^{1}$ Mestranda em Sistemas de Gestão pela Universidade Federal Fluminense (UFF), especialista em Gestão de Empreendimentos Turísticos pela Universidade Federal Fluminense (UFF) e bacharel em Comunicação Social com habilitação em Relações Públicas pela Universidade Federal de Minas Gerais (UFMG).

${ }^{2}$ Orientador. Doutor, mestre e bacharel em Engenharia de Produção pela Universidade Federal do Rio de Janeiro (UFRJ).
}

RC: 84317

Disponível em: https://www.nucleodoconhecimento.com.br/administracao/conhecimentoorganizacional 
ser usadas em processos de gestão do conhecimento e da inovação, assim como identificar os principais tipos de mídias utilizadas e os processos nos quais atuam. A literatura mostrou, ainda, um foco no uso das mídias sociais em processos de compartilhamento de conhecimento.

Palavras-chave: Mídias sociais, Gestão do conhecimento, Compartilhamento de conhecimento.

\section{INTRODUÇÃO}

Uma das problemáticas que vêm se desenhando nas pesquisas recentes sobre Gestão do Conhecimento (GC) é a influência das mídias sociais e de outras tecnologias digitais em seus processos. De acordo com estudos recentes, as mídias sociais podem ser facilitadoras de processos de compartilhamento de conhecimento (BUUNK; SMITH; HALL, 2019; KIM; LEE; OH; 2019; MACEDO et al., 2018; NGAI et al., 2015; PIZZAIA et al., 2018; REZENDE; PEREIRA; OLIVEIRA, 2016); de aprendizagem (KIM; LEE; OH; 2019; MACEDO et al., 2018; NGAl et al., 2015; WINTER; CHAVES, 2017), de criação do conhecimento (PIZZAIA et al., 2018), de gestão de lições aprendidas (WINTER; CHAVES, 2017) e podem dar suporte a comunidades de prática virtuais (LEE et al., 2015; WILBERT et al., 2017), entre outros.

No entanto, para a GC, a compreensão do uso de mídias sociais e de outras ferramentas tecnológicas tem se mostrado desafiadora. De acordo com Macedo (2018), o desafio no estudo dessas mídias "tem sido identificar estratégias para o uso de dados, compartilhamento de conteúdos, extração de informação relevante e produção de conhecimento, a partir da comunicação interativa estabelecida entre os colaboradores de uma organização" (MACEDO et al., 2018, p. 137).

Nesse sentido, o presente artigo tem como objetivo traçar um panorama da pesquisa atual a respeito dos usos das mídias sociais na gestão do conhecimento organizacional. Para tanto, foi realizada, primeiramente, uma contextualização sobre 
gestão do conhecimento com foco em sua dimensão tácita. Posteriormente, foi apresentada uma revisão da literatura que buscou artigos que relacionassem mídias sociais e gestão do conhecimento. A partir desse levantamento, foi possível identificar os principais tipos de mídias utilizadas, assim como os processos nos quais atuam.

\section{UM BREVE CONTEXTO SOBRE GESTÃO DO CONHECIMENTO COM FOCO EM SUA DIMENSÃO TÁCITA}

Embora diferentes modelos estejam presentes na gestão do conhecimento organizacional, sua compreensão pode se fundamentar em duas principais vertentes, que emergiram em diferentes contextos culturais: o processo de criação do conhecimento, observado por Ikujiro Nonaka e Hirotaka Takeuchi (1997) a partir da experiência de companhias japonesas, e suas dinâmicas de conversão do conhecimento tácito e explícito; e as práticas organizacionais que possibilitam a geração, a codificação e a transferência de conhecimento, reconhecidas por Thomas Davenport e Lawrence Prusak (1999) com base em conversas com gerentes de corporações, especialmente dos Estados Unidos (CHOO, 2003).

As organizações são entendidas por Davenport e Prusak (1999) como mercados de conhecimento: enquanto os que buscam conhecimento para resolver um problema são chamados de compradores, os que têm muito conhecimento sobre algo são identificados como vendedores e os que conectam quem precisa e quem tem conhecimento são os corretores. O trabalho dos autores é centrado na captura, na codificação e na transferência de conhecimento, com destaque para o compartilhamento de conhecimento (CHOO, 2003).

Para Nonaka e Takeuchi (1997), o conhecimento não pode ser simplesmente visto e expresso, ou seja, é fundamentalmente "tácito". A essência do trabalho dos autores é de que, portanto, a criação do conhecimento organizacional ocorre através de processos que convertem o conhecimento existente em novos conhecimentos (CHOO, 2003). Ao comparar as epistemologias ocidentais e orientais, os autores RC: 84317

Disponível em: https://www.nucleodoconhecimento.com.br/administracao/conhecimentoorganizacional 
salientam que, ao passo que os cientistas ocidentais entendem a organização como uma máquina que processa informações, numa visão centrada no conhecimento como algo formal e sistemático, ou seja, "explícito", para as companhias japonesas, esse conhecimento é somente a ponta do iceberg.

Com base na distinção apresentada por Michael Polanyi (1966), os autores categorizam o conhecimento em "tácito" e "explícito": "O conhecimento tácito é pessoal, específico ao contexto e, assim, difícil de ser formulado e comunicado. Já o conhecimento explícito ou 'codificado' refere-se ao conhecimento transmissível em linguagem formal e sistemática" (NONAKA; TAKEUCHI, 1997, p. 65). A despeito do foco dos ocidentais no conhecimento explícito e dos japoneses no conhecimento tácito, Nonaka e Takeuchi (1997) compreendem que estes não se excluem mutuamente, mas se complementam, relacionando-se em um processo dinâmico e social, identificado como "conversão do conhecimento": "nosso modelo dinâmico da criação do conhecimento está ancorado no pressuposto crítico de que o conhecimento humano é criado e expandido através da interação social entre o conhecimento tácito e o conhecimento explícito" (NONAKA; TAKEUCHI, 1997, p. 67).

Quatro tipos de conversão do conhecimento são descritos por Nonaka e Takeuchi (1997): na "socialização", o conhecimento tácito é convertido em conhecimento tácito, num processo em que o conhecimento é adquirido por meio de experiências compartilhadas, sem o uso da linguagem; na "externalização", o conhecimento tácito é convertido em conhecimento explícito e a linguagem é utilizada para conceituar práticas apreendidas anteriormente; na "combinação", o conhecimento explícito é convertido em conhecimento explícito e conjuntos de conhecimentos explícitos já existentes são combinados e sistematizados de modo a gerar novos conhecimentos explícitos; e, por fim, na "internalização", o conhecimento explícito é convertido em conhecimento tácito, isto é, know-how técnico e experiências são incorporados ao conhecimento tácito dos indivíduos através de modelos mentais, manuais, 
documentos ou histórias orais. Mais tarde, o conhecimento tácito internalizado é novamente socializado e o processo começa outra vez (NONAKA; TAKEUCHI, 1997).

Espiral do Conhecimento foi o nome dado por Nonaka e Takeuchi (1997) ao processo de criação do conhecimento e seus os quatro tipos de conversão do conhecimento tácito e explícito. De acordo com os autores, cada tipo de conversão do conhecimento separadamente não institui maneiras significativas de criação do conhecimento. Quando conhecimento tácito e explícito interagem é que surge a inovação: "a criação do conhecimento organizacional é um processo em espiral, que começa no nível individual e vai subindo, ampliando comunidades de interação que cruzam fronteiras entre seções, departamentos, divisões e organizações" (NONAKA; TAKEUCHI, 1997, p. 82).

No próximo tópico, trataremos de como a literatura recente vem abordando a questão da utilização das mídias sociais enquanto ferramentas de gestão do conhecimento.

\section{MÍDIAS SOCIAIS E GESTÃO DO CONHECIMENTO ORGANIZACIONAL}

Segundo pesquisas recentes, as mídias sociais vêm sendo usadas de forma satisfatória ou têm potencial para ser usadas em processos de gestão do conhecimento e da inovação, através de plataformas colaborativas diversas como sites de redes sociais, apps, wikis, blogs, microblogs, fóruns, salas de bate-papo, mídias sociais internas, sites de compartilhamento de mídia, sites de social bookmarking, mundos virtuais e webinars (BHIMANI; MENTION; BARLATIER, 2019; BUUNK; SMITH; HALL, 2019; DEY et al., 2019; KIM; LEE; OH; 2019; LEE et al., 2015; LEOPOLD, 2019; MACEDO et al., 2018; MENTION; BARLATIER; JOSSERAND, 2019; NGAl et al., 2015; PIZZAIA et al., 2018; REZENDE; PEREIRA; OLIVEIRA, 2016; WILBERT et al., 2017; WINTER; CHAVES, 2017). Entretanto, percebe-se que, em algumas situações, ocorre um extenso uso de mídias como o Facebook, por exemplo, 
para propósitos pessoais, mas não profissionais, como a produção e o gerenciamento de conhecimento técnico (ALCANTARA; ROCHA; RAMOS, 2015; LEE et al., 2015).

Com base no modelo da Espiral do Conhecimento de Nonaka e Takeuchi (1997), Pizzaia et al. (2018), entendem que uma forma de estimular o compartilhamento de conhecimento e a criação de novos conhecimentos é a formação de grupos no Facebook ou no WhatsApp. De acordo com os autores, essas mídias facilitam a interação, uma vez que permitem o uso de linguagem informal escrita e de recursos multimídia. Os autores ressaltam, ainda, inspirados no mesmo modelo, que o uso das mídias sociais favorece a combinação, isto é, o processo de conversão de conhecimento explícito em explícito. A utilização de mídias sociais como o WhatsApp, por exemplo, para enviar mensagens individuais, auxiliaria ainda o compartilhamento de conhecimento segundo o modelo de von Krogh e Roos (1995), permitindo que o mesmo aconteça "por meio de uma linguagem informal escrita ou verbal e, ainda, o envio de artefatos multimídia como fotos e vídeos, evitando assim ruídos ideológicos e distorções no aprendizado individual e organizacional" (PIZZAIA et al., 2018, p. 74). No modelo, classifica-se o aprendizado em individual - alicerçado nas relações e nas comunicações para o processamento de dados das informações recebidas - e organizacional - embasado em redes auto-organizadas formadas por relações comunicacionais (PIZZAIA et al., 2018).

Ao pesquisar um grupo do WhatsApp constituído por profissionais da área de tecnologia, Macedo et al. (2018) também perceberam que é possível o uso dessa ferramenta e outras tecnologias da informação e da comunicação (TICs) em processos de gestão do conhecimento. De acordo com os autores, ao possibilitar discussões entre especialistas, as plataformas sociais on-line promovem a inteligência coletiva, permitem que o conhecimento tácito e pessoal seja visto e acessado e diminuem o tempo e os esforços necessários. Foram, assim, destacadas como importantes facilitadoras de compartilhamento de conhecimento tácito, envolvendo 
processos de aprendizagem, de compartilhamento de experiências, de solução de problemas e de inovação.

Ademais, Buunk, Smith e Hall (2019) identificaram pelo menos três aspectos do conceito de Ba, como formulado por Nonaka e Konno (1998), em uma plataforma virtual que oferece recursos de mídias sociais e permite o compartilhamento de conhecimento entre trabalhadores da administração pública na Escócia. Conforme os autores, Ba pode ser definido como "um espaço contextual que é compartilhado com outras pessoas, e dentro do qual o compartilhamento, a transferência ou a mobilização de conhecimento podem ocorrer" (BUUNK; SMITH; HALL, 2019, p. 1135, tradução nossa). Apesar disso, destacam que é imprescindível que o tema do uso de mídias sociais para o compartilhamento de conhecimento no setor público seja explorado mais a fundo.

Em contextos como o da gestão de projetos, em que há um isolamento entre os gerentes, os trabalhadores que realizam as atividades do projeto e a equipe gestora, sem que os gerentes interajam entre si, um importante papel pode ser exercido pelas comunidades de prática (LEE et al., 2015), que podem ser definidas como um "sistema de atividades no qual participantes compartilham entendimentos sobre o que se está fazendo, e o que isso significa para suas vidas e para a comunidade" (LAVE; WENGER, 1991, p. 98, tradução nossa). Ao facilitar a interação entre os gerentes, contribuindo para sua aproximação e reforçando laços, essas plataformas tornariam possível a recuperação de um sentimento de camaradagem entre eles, resultando numa melhor eficácia da gestão de projetos. Contudo, apesar desse potencial, o que se percebe é que ainda há uma baixa utilização das mídias sociais por gerentes de projetos para fins profissionais e para estimular comunidades de prática, fazendo com que representem recursos inexplorados pelas organizações e pela pesquisa (LEE et al., 2015). Em outros ambientes, notou-se que comunidades de prática virtuais (VCoPs, do inglês Virtual Communities of Practice) têm potencial para auxiliar processos de inovação (WILBERT et al.; 2017).

Disponível em: https://www.nucleodoconhecimento.com.br/administracao/conhecimentoorganizacional 
Ao pesquisar a plataforma virtual de gestão do conhecimento de uma organização, Rezende, Pereira e Oliveira (2016) investigaram de que forma uma ferramenta tecnológica pode operar como contexto capacitante, ou seja, um ambiente e artefatos favoráveis à disseminação do conhecimento "que sejam capazes de mobilizar indivíduos de maneira a que ativem seus excedentes cognitivos e proporcionem diferenciais para as organizações" (REZENDE; PEREIRA; OLIVEIRA, 2016, p. 73). Os pesquisadores puderam confirmar a ativação de excedentes cognitivos dos funcionários, o que faz com que a plataforma seja usada para gerar valor em processos, produtos e serviços. A literatura citou, ainda, ferramentas colaborativas como as wikis, por exemplo, como plataformas que dão suporte à gestão de lições aprendidas (GLA), contribuindo para a aprendizagem organizacional, tornando os projetos mais produtivos, os funcionários mais colaborativos e facilitando a disseminação do conhecimento (WINTER; CHAVES, 2017).

É importante pontuar a influência das mídias sociais em processos de gestão do conhecimento também no ambiente externo, ainda que este trabalho esteja focado em como as mídias sociais contribuem para processos de gestão do conhecimento dentro das organizações. Em contextos em que as empresas oferecem poucos recursos de suporte ao consumidor, Dey et al. (2019) perceberam que as mídias sociais podem ajudar em processos de cocriação de conhecimento. Essas mídias auxiliam levando inovação, habilidades, conhecimentos e experiências do nível micro para o domínio público mais amplo e oferecendo uma plataforma para aprendizagem colaborativa, criação e disseminação dessas habilidades, conhecimentos e experiências. Ao estudar a economia colaborativa ou economia compartilhada, Kalil (2018) descreve como as mídias sociais permitem a existência de um mercado baseado em compartilhamentos. Notou-se também que há uma relação entre características individuais, intenção de compartilhar conhecimento e compartilhamento de conhecimento ao pesquisar comunidades de aprendizagem por meio de grupos no Facebook (KIM; LEE; OH, 2019). 
Quatro tipos principais de mídias sociais usadas pelas organizações em atividades de inovação foram encontradas: "mídias sociais públicas", como Facebook, Twitter, YouTube, Linkedln, etc.; "mídias sociais criadas pelas organizações", que são plataformas com recursos de mídias sociais, desenvolvidas internamente e usadas para interação com os públicos internos e externos; "mídias sociais licenciadas para as organizações", que são ferramentas desenvolvidas externamente e contratadas para funcionar como plataformas de redes sociais corporativas; e, por fim, "mídias sociais intermediárias de inovação", usadas na criação de serviços personalizados em inovação (BHIMANI; MENTION; BARLATIER, 2019).

A revisão da literatura sobre a utilização de mídias sociais na gestão do conhecimento pode ser resumida no Quadro 1. O Quadro correlaciona plataformas e ferramentas de comunicação digital com processos de GC, de acordo com o que foi levantado na literatura recente.

Quadro 1 - Tipos de mídias sociais utilizadas na gestão do conhecimento organizacional e os processos nos quais atuam

\begin{tabular}{|l|l|l|}
\hline Tipos de mídias sociais & Processos & Referência \\
\hline Grupos no Facebook & $\begin{array}{l}\text { Produção } \\
\text { gestão } \\
\text { conhecimento de } \\
\text { técnico }\end{array}$ & ALCANTARA; ROCHA; RAMOS, \\
\hline $\begin{array}{l}\text { Mídias sociais de uma } \\
\text { maneira geral }\end{array}$ & $\begin{array}{l}\text { Gestão } \\
\text { inovação da }\end{array}$ & BHIMANI; \\
\hline $\begin{array}{l}\text { KnowledgeHub (plataforma } \\
\text { de colaboração on-line para }\end{array}$ & $\begin{array}{l}\text { Compartilhamento } \\
\text { de conhecimento }\end{array}$ & BUUNK; SMITH; HALL, 2019 \\
$\begin{array}{l}\text { o setor público, oferecida } \\
\text { gratuitamente tanto a }\end{array}$ & tácito & \\
\hline
\end{tabular}

Disponível em: https://www.nucleodoconhecimento.com.br/administracao/conhecimentoorganizacional 


\begin{tabular}{|c|c|c|}
\hline $\begin{array}{l}\text { indivíduos quanto a } \\
\text { comunidades de prática) }\end{array}$ & & \\
\hline $\begin{array}{l}\text { Grupos no Facebook, IRC } \\
\text { Chat Rooms (salas de bate- } \\
\text { papo) e fóruns de } \\
\text { discussão em blogs }\end{array}$ & $\begin{array}{l}\text { Cocriação de } \\
\text { conhecimento }\end{array}$ & DEY et al., 2019 \\
\hline Grupos no Facebook & $\begin{array}{l}\text { Compartilhamento } \\
\text { de conhecimento } \\
\text { em comunidades } \\
\text { de aprendizagem }\end{array}$ & KIM; LEE; OH; 2019 \\
\hline $\begin{array}{l}\text { Diversas ferramentas } \\
\text { comunicação digital, com } \\
\text { destaque para o Linkedln, } \\
\text { webinars, fóruns de } \\
\text { discussão e mídias sociais } \\
\text { internas }\end{array}$ & $\begin{array}{l}\text { Compartilhamento } \\
\text { de conhecimento } \\
\text { em comunidades } \\
\text { de prática }\end{array}$ & LEE et al., 2015 \\
\hline $\begin{array}{l}\text { Mídias sociais de uma } \\
\text { maneira geral }\end{array}$ & $\begin{array}{l}\text { Gestão da } \\
\text { inovação }\end{array}$ & LEOPOLD, 2019 \\
\hline Grupo no WhatsApp & $\begin{array}{l}\text { Compartilhamento } \\
\text { de conhecimento } \\
\text { e aprendizagem }\end{array}$ & MACEDO et al., 2018 \\
\hline $\begin{array}{l}\text { Mídias sociais de uma } \\
\text { maneira geral }\end{array}$ & $\begin{array}{l}\text { Gestão da } \\
\text { inovação }\end{array}$ & $\begin{array}{l}\text { MENTION; } \quad \text { BARLATIER; } \\
\text { JOSSERAND, } 2019\end{array}$ \\
\hline $\begin{array}{l}\text { Blogs / microblogs e } \\
\text { comunidades virtuais / on- } \\
\text { line }\end{array}$ & $\begin{array}{l}\text { Compartilhamento } \\
\text { de conhecimento }\end{array}$ & NGAl et al., 2015 \\
\hline $\begin{array}{l}\text { Sites de compartilhamento } \\
\text { de mídia, sites de social } \\
\text { bookmarking, sites de }\end{array}$ & Aprendizagem & \\
\hline
\end{tabular}

RC: 84317

Disponível em: https://www.nucleodoconhecimento.com.br/administracao/conhecimentoorganizacional 


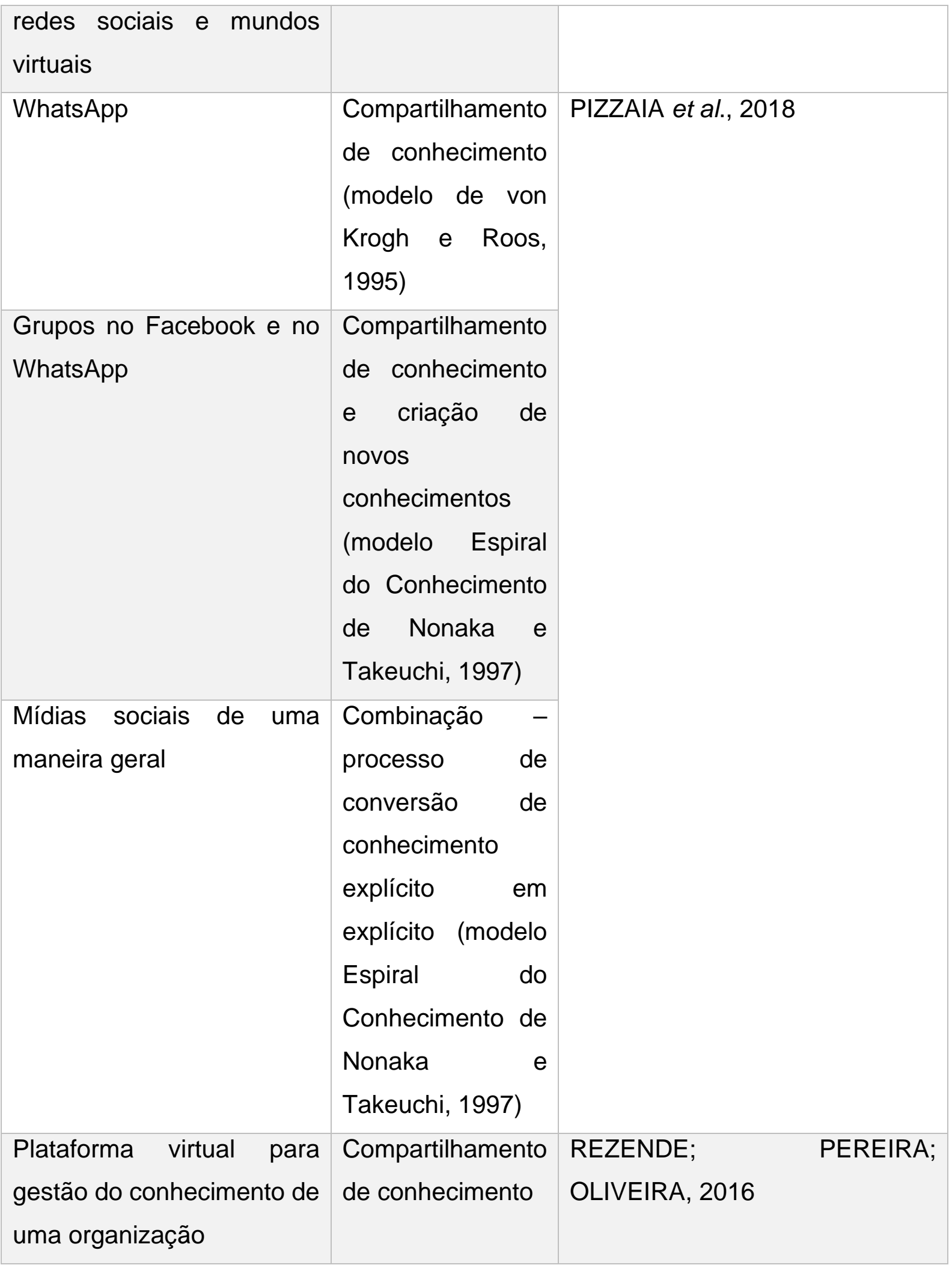

RC: 84317

Disponível em: https://www.nucleodoconhecimento.com.br/administracao/conhecimentoorganizacional 


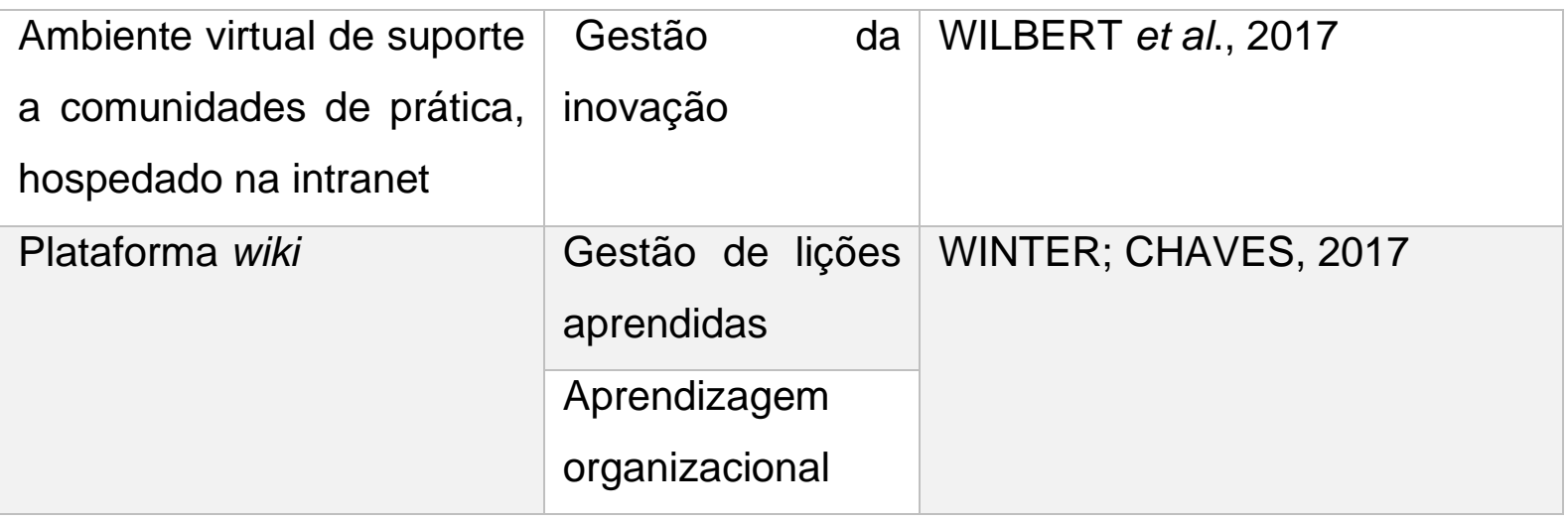

Fonte: Elaborado pelos autores

A partir da análise do quadro, é possível inferir que a maioria das mídias sociais utilizadas para a gestão do conhecimento, de acordo com as pesquisas levantadas, atua em processos de compartilhamento de conhecimento. $O$ fato de 0 compartilhamento estar presente em todos os modelos de gestão do conhecimento $\mathrm{e}$ qualquer tipo de compartilhamento se dar por meio de algum tipo de comunicação (PIZZAIA et al., 2018) pode ser uma possível explicação para isso. A aprendizagem é outro processo frequentemente mencionado, assim como a gestão da inovação, se constituindo em atividades que podem ser fomentadas pela utilização de mídias sociais.

\section{CONSIDERAÇÕES FINAIS}

A literatura recente tem se preocupado com a questão do uso de mídias sociais e outras tecnologias digitais na gestão do conhecimento, porém sua compreensão ainda se mostra desafiadora (MACEDO et al., 2018). O presente artigo teve como objetivo, portanto, traçar um panorama atual da pesquisa acerca do uso de mídias sociais na gestão do conhecimento por meio de uma revisão da literatura sobre o tema em artigos recentes publicados em periódicos nacionais e internacionais.

A análise dos artigos levantados nos permite concluir que as mídias sociais têm sido utilizadas com sucesso ou têm potencial para ser utilizadas em processos de gestão

RC: 84317

Disponível em: https://www.nucleodoconhecimento.com.br/administracao/conhecimentoorganizacional 
do conhecimento e da inovação. Percebe-se, ainda, que há um foco na utilização das mídias sociais em processos de compartilhamento de conhecimento. Aprendizagem e inovação também foram bastante citadas, de forma secundária.

Pesquisas futuras podem explorar outros aspectos, como, por exemplo, as características das mídias utilizadas na gestão do conhecimento e de seus usuários, assim como aprofundar a compreensão do fenômeno por meio da coleta e da análise de dados empíricos. Um desafio que vem se apresentando é o fato de que as mídias sociais também produzem ruídos na gestão do conhecimento organizacional, como vem ficando claro na produção das fake news na sociedade como um todo. Uma literatura cada vez mais intensa vem tratando deste fenômeno, talvez ainda predominantemente no âmbito da sociedade, mas chegará o momento em que a gestão deverá cuidar disso com mais atenção.

\section{REFERÊNCIAS}

ALCANTARA, Sonia Regina Amorim Soares de; ROCHA, Thays Lyanny da Cunha Garcia; RAMOS, Lilian. Análise da utilização do Facebook na produção de conhecimento técnico por profissionais do secretariado executivo. Revista Expectativa, v. 14, n. 14, 2015. Disponível em: http://erevista.unioeste.br/index.php/expectativa/article/view/12239. Acesso em: 11 mar. 2021.

BHIMANI, Hardik; MENTION, Anne-Laure; BARLATIER, Pierre-Jean. Social media and innovation: A systematic literature review and future research directions. Technological Forecasting and Social Change, v. 144, p. 251-269, jul. 2019. Disponível em: https://www.sciencedirect.com/science/article/abs/pii/S004016251 Acesso em: 11 mar. 2021.

BUUNK, Iris; SMITH, Colin F.; HALL, Hazel. Tacit knowledge sharing in online environments: Locating 'Ba' within a platform for public sector professionals. Journal

RC: 84317

Disponível em: https://www.nucleodoconhecimento.com.br/administracao/conhecimentoorganizacional 
of Librarianship and Information Science, v. 51, n. 4, p. 1134-1145, dez. 2019. Disponível em: https://journals.sagepub.com/doi/abs/10.1177/0961000618769982. Acesso em: 11 mar. 2021.

$\mathrm{CHOO}$, Chun Wei. Perspectives on Managing Knowledge in Organizations. Cataloging \& Classification Quarterly, v. 37, n. 1-2, p. 205-220, jul. 2003b. Disponível em: https://www.tandfonline.com/doi/abs/10.1300/J104v37n01_14. Acesso em: 12 abr. 2021.

DAVENPORT, Thomas; PRUSAK, Lawrence. Conhecimento empresarial. São Paulo: Publifolha, 1999.

DEY, Bidit Lal; SARMA, Meera; PANDIT, Ameet; SARPONG, David; KUMARI, Sushma; PUNJAISRI; Khanyapuss. Social media led co-creation of knowledge in developing societies: SME's roles in the adoption, use and appropriation of smartphones in South Asia. Production Planning \& Control, v. 30, n. 10-12, p. 1019-1031, 10 set. 2019.2 Disponível em: https://www.tandfonline.com/doi/abs/10.1080/09537287.2019.1582106?journalCode =tppc20. Acesso em: 11 abr. 2021.

KALIL, Mateus Oliveira; MARAVILHAS-LOPES; Sérgio Paulo. O compartilhamento de informação na construção de uma economia colaborativa e geração de modelos de negócios inovadores. Perspectivas em Gestão \& Conhecimento, v. 8, n. 3, p. 110 126, 26 dez. 2018.2 Disponível em: https://periodicos.ufpb.br/ojs2/index.php/pgc/article/view/34694. Acesso em: $11 \mathrm{abr}$. 2021.

KIM, Hannah; LEE, Jeongmin; OH, Sung Eun. Individual characteristics influencing the sharing of knowledge on social networking services: online identity, self-efficacy, and knowledge sharing intentions. Behaviour \& Information Technology, p. 1-12, 9 abr. 2019. 
https://www.tandfonline.com/doi/abs/10.1080/0144929X.2019.1598494 journalCode=tbit20. Acesso em: 11 abr. 2021.

LAVE, Jean; WENGER, Etienne. Situated learning: legitimate peripheral participation. New York: Cambridge University Press, 1991.

LEE, Lorraine; REINICKE, Bryan; SARKAR, Robin; ANDERSON, Rita. Learning through Interactions: Improving Project Management through Communities of Practice. Project Management Journal, v. 46, n. 1, p. 40-52, fev. 2015. Disponível em: https://journals.sagepub.com/doi/10.1002/pmj.21473. Acesso em: 11 abr. 2021.

LEOPOLD, Helmut. Social media and corporate innovation management-Eight rules to form an innovative organisation. e \& i Elektrotechnik und Informationstechnik, v. 136, n. 3, p. 241-253, jun. 2019. Disponível em: https://www.springerprofessional.de/en/social-media-and-corporate-innovationmanagement-eight-rules-to-/16824768. Acesso em: 11 abr. 2021.

MACEDO, Valeria; DANTAS, Daniele Cristina; GUEDES, Rodrigo Duarte; CAVALCANTI, Marcos do Couto Bezerra. O uso do aplicativo Whatsapp nas práticas de gestão do conhecimento: o caso de uma comunidade virtual informal de profissionais na área de tecnologia. Perspectivas em Gestão \& Conhecimento, João Pessoa, v. 8, número especial, p. 135-150, out. 2018. Disponível em: https://periodicos.ufpb.br/ojs2/index.php/pgc/article/view/42241. Acesso em: 11 abr. 2021.

MENTION, A.-L.; BARLATIER, Pierre-Jean; JOSSERAND, Emmanuel. Using social media to leverage and develop dynamic capabilities for innovation. Technological Forecasting and Social Change, v. 144, p. 242-250, jul. 2019. Disponível em: https://www.sciencedirect.com/science/article/abs/pii/S0040162519304561. Acesso em: 11 abr. 2021. 
NGAI, Eric W.T.; MOON, Ka-leung Karen; LAM, S.S.; CHIN, Eric S. K.; TAO, Spencer S.C. Social media models, technologies, and applications: An academic review and case study. Industrial Management \& Data Systems, v. 115, n. 5, p. 769-802, 8 jun. 2015. Disponível em: https://www.emerald.com/insight/content/doi/10.1108/IMDS-032015-0075/full/html. Acesso em: 11 abr. 2021.

NONAKA, Ikujiro; KONNO, Noboru. The concept of "Ba': Building foundation for Knowledge Creation. California Management Review, V. 40, n. 3, p. 40-54, 1998. Disponível em: https://journals.sagepub.com/doi/10.2307/41165942. Acesso em: 11 abr. 2021.

NONAKA, Ikujiro; TAKEUCHI, Hirotaka. Criação do Conhecimento na Empresa: como as empresas geram a dinâmica da inovação. Rio de Janeiro: Campus, 1997.

PIZZAIA, Angela; PEGINO, Paulo Marcelo Ferraresi; COLLA, Júlio Ernesto; TENÓRIO, Nelson. O papel da comunicação na gestão do conhecimento: aspectos relevantes e estímulo a novas pesquisas. Perspectivas em Gestão \& Conhecimento, João Pessoa, v. 8, n. 2, p. 62-81, maio/ago. 2018. Disponível em: https://periodicos.ufpb.br/ojs2/index.php/pgc/article/view/33522. Acesso em: 11 abr. 2021.

POLANYI, Michael. The tacit dimension. London: Routdedge \& Kegan Paul, 1966.

REZENDE, José Francisco; PEREIRA, Rafael Deolindo; OLIVEIRA, Danielle Almeida. Plataformas para gestão do conhecimento: estudo de caso sobre a ativação do valor de excedentes cognitivos por meio do desenvolvimento de um contexto capacitante virtual. Perspectivas em Gestão \& Conhecimento, v. 6, n. 1, p. 72-88, 6 jun. 2016. Disponível em: https://periodicos.ufpb.br/ojs/index.php/pgc/article/view/26312. Acesso em: 11 abr. 2021.

VON KROGH, Georg; ROOS, Johan. Organizational Epistemology. New York: St. Martin's Press, 1995.

RC: 84317

Disponível em: https://www.nucleodoconhecimento.com.br/administracao/conhecimentoorganizacional 
WILBERT, Julieta Kaoru Watanabe; OLIVEIRA, Isabela Cristini Gonçalves de; STEIL, Andrea Valéria; DANDOLINI, Gertrudes Aparecida; SOUZA, João Artur de. O uso de comunidades de prática virtuais (VCoPs) para fins de inovação segundo percepção de seus membros. Perspectivas em Gestão \& Conhecimento, João Pessoa, v. 7, Número Especial, p. 109-125, mar. 2017. Disponível em: https://periodicos.ufpb.br/ojs2/index.php/pgc/article/view/33055. Acesso em: $11 \mathrm{abr}$. 2021.

WINTER, Roberto; CHAVES, Marcirio Silveira. Innovation in the management of lessons learned in an IT project with the adoption of social media. International Journal of Innovation, v. 5, n. 2, p. 156-170, ago. 2017. Disponível em: https://periodicos.uninove.br/innovation/article/view/9829. Acesso em: 11 abr. 2021.

Enviado: Abril, 2021.

Aprovado: Maio, 2021. 